Proceedings of the WELCOME Scientific Meeting on Hybrid Nanostructures, Torun, Poland, August 28-31, 2011

\title{
Atomistic Calculation of Coulomb Interactions in Semiconductor Nanocrystals: Role of Surface Passivation and Composition Details
}

\author{
M. Chwastyk, P. Różanski And M. Zieliński* \\ Instytut Fizyki UMK, Grudziądzka 5, 87-100 Toruń, Poland
}

\begin{abstract}
We report a theoretical investigation of electronic properties of semiconductor InAs and GaAs nanocrystals. Our calculation scheme starts with the single particle calculation using atomistic tight-binding model including spin-orbital interaction and $d$-orbitals. Then the exciton binding energies are calculated with screened Coulomb interaction. We study the role of surface passivation effects by varying value of surface passivation potential. We compare results obtained with dot center positioned on different lattice sites thus containing different number of anion and cations. We conclude that passivation of surface states affects significantly single particle energies and the value of electron-hole Coulomb attraction. Interestingly, due to limited screening, the short-range (on-site) contribution to the electron-hole Coulomb attraction plays significant role for small nanocrystals with radius smaller than $1 \mathrm{~nm}$.
\end{abstract}

PACS: 73.21.La, 78.67.Bf, 78.67.Hc, 73.20.Fz, 71.15.-m

\section{Introduction}

Continuous matter approaches demonstrated their usability in describing main spectral features of semiconductor quantum dots $[1,2]$ including chemically synthesized nanocrystals $[3,4]$, yet the effective mass approximation (EMA) overestimates single particle gap [5] or electron-hole binding energy [6], while atomistic approaches have proven to be necessary for accurate description of the details of electronic and excitonic spectra of semiconductor nanocrystals [4, 7-11].

In this paper we adopt the tight binding approach which accounts for effects of dot size, shape or composition on the atomic scale with a modest computational effort even for large nanosystems. We use $s p^{3} d^{5} s^{*}$ parameterization [12, 13] including spin-orbit effects [14] and $d$-orbitals both playing important role in description of nanocrystals spectra, especially for small size systems $[8,9]$. Recent research has proven that passivation of surface dangling bonds plays important role for electronic and optical properties of nanocrystals affecting nanocrystal gap [15-18]. In this work we mimic passivation by shifting energies of dangling bonds (unsaturated hybrid orbitals) [19] and effectively removing the dangling bonds states from the single particle gap region. This approach has the advantage of not increasing the Hamiltonian size, while the explicit modeling of surface passivation leads to a larger Hamiltonian dimension and finally more demanding computation. By varying the value of dangling bond shift (or effectively "surface passivation potential") within wide $-20 \ldots 20 \mathrm{eV}$ range, we simulate effects of different passivating agents.

Single particle states obtained in the TB step of calcu-

* corresponding author; e-mail: mzielin@fizyka.umk.pl lation are later used for calculation of the Coulomb matrix elements $[13,20]$. In this work we focus our attention on electron ground state $(e 1)$-hole ground state $(h 1)$ direct Coulomb integral to estimate exciton binding. Here, we ignore the exchange interactions and corrections due to correlations, which are much smaller than the direct Coulomb interactions, and approximated exciton binding energy with single screened Coulomb integral [13]:

$$
\begin{aligned}
& J_{e_{1} h_{1}}=\iint \phi_{e_{1}}^{*}\left(\boldsymbol{r}_{1}\right) \phi_{h_{1}}^{*}\left(\boldsymbol{r}_{2}\right) \frac{e^{2}}{\varepsilon\left(\boldsymbol{r}_{1}, \boldsymbol{r}_{2}\right)\left|\boldsymbol{r}_{1}-\boldsymbol{r}_{2}\right|} \\
& \times \phi_{h_{1}}\left(\boldsymbol{r}_{2}\right) \phi_{e_{1}}\left(\boldsymbol{r}_{1}\right),
\end{aligned}
$$

where $\varepsilon\left(\boldsymbol{r}_{1}, \boldsymbol{r}_{2}\right)$ is the position-dependent dielectric constant, and $\phi$ are single-particle wave functions obtained by diagonalization of the TB Hamiltonian and are given as linear combinations of atomic orbitals (LCAO):

$$
\phi_{i}=\sum_{\boldsymbol{R}, \alpha} c_{\boldsymbol{R} \alpha}^{i}|\boldsymbol{R} \alpha\rangle .
$$

Substituting $\phi$ in LCAO form into the formula (1), one obtains fourfold summation over the Coulomb and exchange integrals calculated in atomic basis [13, 21]. Then, by series of approximations, including only monopolemonopole terms of two-center Coulomb integrals, one obtains

$$
\begin{aligned}
& J_{e_{1} h_{1}} \approx \sum_{\boldsymbol{R}_{i}, \boldsymbol{R}_{j}} V\left(\boldsymbol{R}_{i}-\boldsymbol{R}_{j}\right)\left(\sum_{\alpha_{1}} c_{\boldsymbol{R}_{i} \alpha_{1}}^{e_{1} *} c_{\boldsymbol{R}_{i} \alpha_{1}}^{e_{1}}\right) \\
& \quad \times\left(\sum_{\alpha_{2}} c_{\boldsymbol{R}_{j} \alpha_{2}}^{h_{1} *} c_{\boldsymbol{R}_{j} \alpha_{2}}^{h_{1}}\right) \\
& =\sum_{\boldsymbol{R}_{i}, \boldsymbol{R}_{j}} V\left(\boldsymbol{R}_{i}-\boldsymbol{R}_{j}\right)\left(\sum_{\alpha_{1}}\left|c_{\boldsymbol{R}_{i} \alpha_{1}}^{e_{1}}\right|^{2}\right) \\
& \quad \times\left(\sum_{\alpha_{2}}\left|c_{\boldsymbol{R}_{j} \alpha_{2}}^{h_{1}}\right|^{2}\right),
\end{aligned}
$$


where:

$$
\begin{aligned}
& V\left(\boldsymbol{R}_{i}-\boldsymbol{R}_{j}\right) \equiv v_{i j} \\
& \quad= \begin{cases}\frac{e^{2}}{\varepsilon\left(\boldsymbol{R}_{i}, \boldsymbol{R}_{j}\right)\left|\boldsymbol{R}_{i}-\boldsymbol{R}_{j}\right|} & \text { if } \boldsymbol{R}_{i} \neq \boldsymbol{R}_{j}, \\
U_{\text {at }} & \text { if } \boldsymbol{R}_{i}=\boldsymbol{R}_{j},\end{cases}
\end{aligned}
$$

and on-site $\left(\boldsymbol{R}_{i}=\boldsymbol{R}_{j}\right)$ integrals have been approximated with single $U_{\text {at }}$ that depends only on atom located at $\boldsymbol{R}_{i}$.

For calculation of unscreened electron-hole Coulomb integral we use on-site (bare atomic) $U_{\text {at }}$ values $U_{\text {In }}=$ $7.16 \mathrm{eV}, U_{\mathrm{Ga}}=8.3 \mathrm{eV}$ and $U_{\mathrm{As}}=10.6 \mathrm{eV}$ obtained by an approach analogous to that of Ref. [22] and set $\varepsilon\left(\boldsymbol{R}_{i}, \boldsymbol{R}_{j}\right)=1$ in the off-site $\left(\boldsymbol{R}_{i}=\boldsymbol{R}_{j}\right)$ contribution. For calculation of screened electron-hole Coulomb integral, the off-site terms are screened with bulk dielectric constant $\left(\varepsilon_{\mathrm{GaAs}}=12.9, \varepsilon_{\mathrm{InAs}}=15.15\right)$, while screening of on-site terms is limited [19,21] and we use $1 / 3$ of free atom $U_{\text {at }}$ for screened on-site terms [4]. This approach is justified by the fact that screening (the Thomas-Fermi) radius $(\approx 2-4 \AA)$ is on the order of bond length $[19,21]$ resulting in nearly bulk screening of off-site (long-range) terms and limited screening of on-site (short-range) terms contribution.

In principle, the full treatment of screened Coulomb interaction should account for surface polarization effects [23-25], i.e. formation of image charges due to dielectric constant discontinuity at the quantum dot interface. However, it has been shown [22] that there is nearly perfect cancellation of the surface polarization contribution to electron-hole Coulomb integral, with self-energy corrections to single particle electron and hole states. Thus, exciton energy (neglecting correlation and exchange effects) can be approximated as

$$
E_{\mathrm{X}} \approx E_{\text {gap }}^{s p}-J_{e_{1} h_{1}},
$$

where $E_{\text {gap }}^{s p}=E_{1}-H_{1}$ is quantum dot single particle (HOMO-LUMO) gap taken from TB calculation and $J_{e_{1} h_{1}}$ is screened Coulomb interaction given by formula (3), with $e_{1}$ and $h_{1}$ being electron and hole ground states, as well as $E_{1}$ and $H_{1}$ being corresponding eigenenergies.

\section{Single particle gap}

Figure 1a shows evolution of the single particle gap $E_{\text {gap }}$ calculated for InAs and GaAs spherical nanocrystals as a function of nanocrystal radius. Lines and symbols correspond to the dot center located on anion, cation, and the "midpoint" being average position of the both. Overall shape of quantum dot is spherical, yet the actual symmetry is tetrahedral $\left(T_{d}\right)$ and choosing different origin one obtains structures differing with number of anion and cations. The effect is small for nanocrystals larger than $1.5 \mathrm{~nm}$, however it is not negligible for smaller nanocrystal with maximum spread of the gap value $\pm 0.2 \mathrm{eV}$ for $r=0.6 \mathrm{~nm}$ InAs nanocrystals. It should be noted that even for largest considered ( $>2.5 \mathrm{~nm}$ ) nanocrystals, the single particle gap is still much larger than the

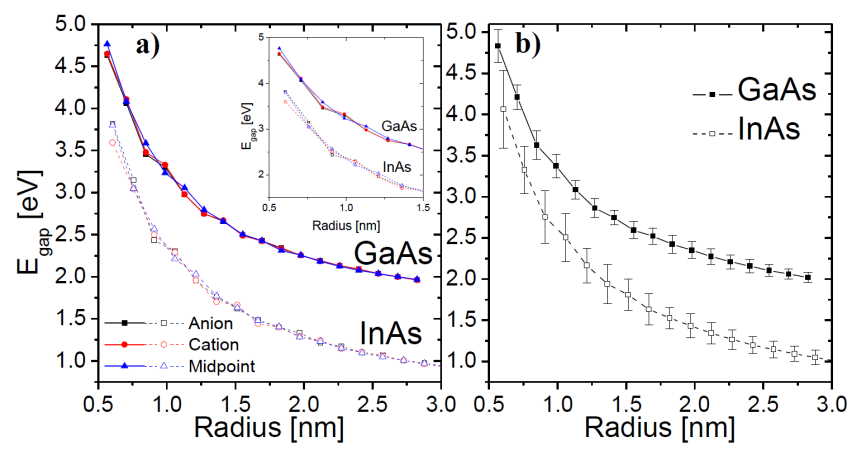

Fig. 1. Evolution of the single particle gap $E_{\text {gap }}$ calculated for InAs and GaAs spherical nanocrystals as a function of nanocrystal radius. Lines and symbols correspond to (a) the dot center located on anion, cation, and the "midpoint" (b) extreme values of surface passivation potential $-20 \ldots 20 \mathrm{eV}$ (description in text).

asymptotic bulk value and that generally in an atomistic calculation the single-particle energy gap scales slower than $1 / R^{2}$ rule predicted by simple particle-in a box model $[6,8]$.

Apart from composition details variations, one can calculate TB spectra using different values of surface passivation potential (dangling bond shift), thus mimicking effects of different passivating factors [26]. Figure 1b shows evolution of the single particle gap $E_{\text {gap }}$ calculated for InAs and GaAs spherical nanocrystals as a function of nanocrystal radius, averaged over results obtained for different, extreme values of surface passivation potential $-20,20 \mathrm{eV}$ and different dot center location (anion, cation, midpoint). Error bars in Fig. 1b mark extreme results obtained for different calculations. We notice that uncertainties due to surface passivation dominate over those related to anion/cation composition details. We also noticed that uncertainties due to composition details do not simply add up to uncertainties due to surface passivation potential variation. This is because differently centered dots have different number of surface anions/ cations (surface composition) and correspondingly different number of (single or double) passivated dangling bonds, belonging to different ionic species.

For large band GaAs nanocrystals gap uncertainties reach $\approx 0.15 \mathrm{eV}$, about $4 \%$ of the single particle gap value and are about $\approx 0.06 \mathrm{eV}$ (or $3 \%$ of $E_{\text {gap }}$ ) for larger nanocrystals, rather constant value over wide range of different sizes. Interestingly, for small band gap InAs nanocrystals the effect of different choice of surface passivation potential is more pronounced, with error bars reaching $\approx 0.3 \mathrm{eV}$, or up to $12 \%$ of the single particle gap and $\approx 0.08 \mathrm{eV}$ ( $8 \%$ of $E_{\text {gap }}$ ) even for largest considered InAs nanocrystals.

Such large dependence of single particle gap on surface passivation, suggests that different passivation agents can be used for tailoring nanocrystal band gap for certain optical applications in agreement with recent experiments. On the contrary, nanocrystals built from large band gap bulk material have more stable single particle gap with 
respect to surface passivation effect, which can be more useful for different applications.

In Fig. 2a we plot evolution of single particle band gap as function dangling bond shift for several GaAs nanocrystals. Figure 2a confirms our speculations that effect of surface passivation depends on nanocrystal diameter in the way the surface/volume ratio does.

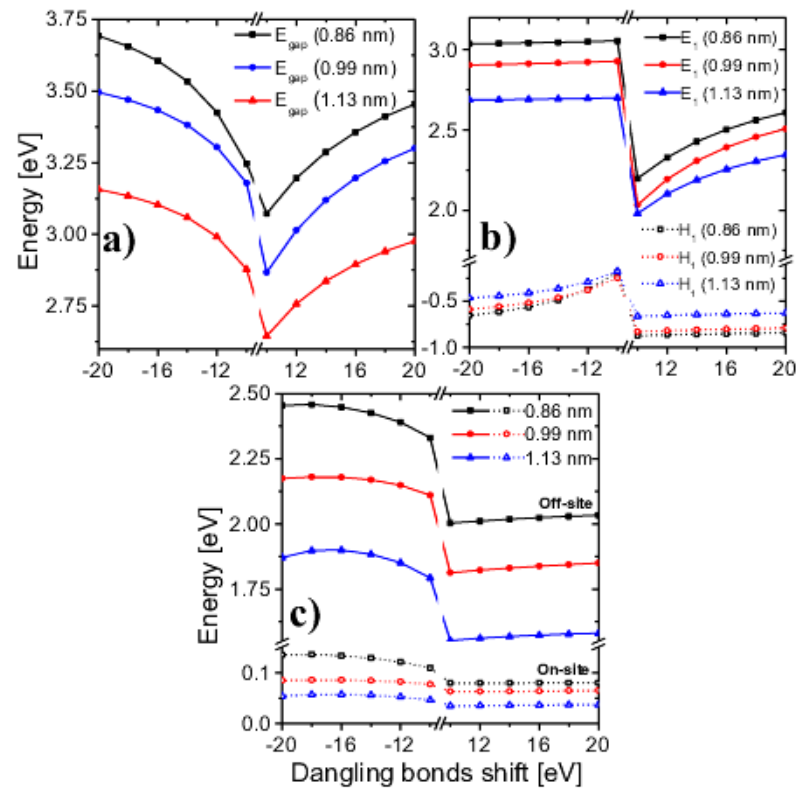

Fig. 2. (a) Evolution of the single particle gap $E_{\text {gap }}$, (b) electron and hole ground state energies and (c) electron-hole unscreened $J_{e_{1} h_{1}}$ Coulomb integral as function of dangling bond shift for several GaAs nanocrystals.

There is significant reduction of $E_{\text {gap }}$ for dangling bond shift within $-10 \ldots 10 \mathrm{eV}$ range. For even smaller $(-5 \ldots 5 \mathrm{eV}$ not shown in Fig. 2) shift values the surface states originating from dangling bonds enter the gap region effectively leading to electron-hole recombination through surface state or in other words no passivation at all. For passivation larger than $\pm 10 \mathrm{eV}$ surface states are effectively decoupled from "volume" quantum dot states, however the evolution of single particle gap is far from being converged despite large dangling bond shifts.

It should be noted that even though surface states due to dangling bonds are shifted away from the gap region, yet the value of the dangling bond shift acts also as effective potential added to surface atoms, thus changing overall confinement potential. Applying large dangling bond shift effectively separates volume states form surface both in spectral and spatial terms, thus effectively "squeezing" confined states and resulting in increase of single particle energies. Interestingly, surface passivation with large, negative values of dangling bond shift [26] leads to $\approx 0.2 \mathrm{eV}$ larger single particle band gap than that for large, positive passivation.

In Fig. 2b we plot evolution of electron and hole ground state as function surface passivation potential for several
GaAs nanocrystals. We find that for large negative (positive) shifts, electron (hole) states do not change with the choice significantly with dangling bond shift. Thus the coupling between surface and volume states is effective only between one type of carriers at a time, depending on the choice of dangling bond shift sign, while the other carrier type is effectively decoupled from the surface. We point here however that accurate modeling of surface passivation would demand a rigorous modeling of surface ad-atoms, preferably using ab initio approach.

\section{Unscreened electron-hole interaction}

Figure 3 shows unscreened Coulomb integral (with electron and hole occupying their lowest single particle $s$ states) for (a) InAs and (b) GaAs nanocrystals as a function of nanocrystal radius, calculated using TB wave function and, for comparison, calculated by EMA [27].

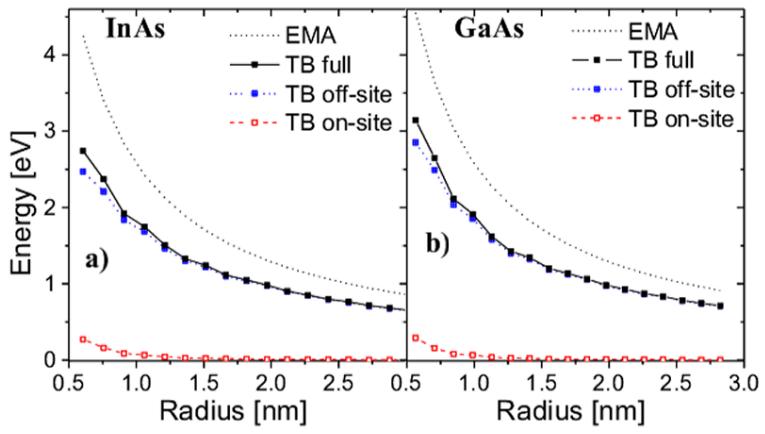

Fig. 3. Unscreened electron-hole $J_{e_{1} h_{1}}$ Coulomb integral for (a) InAs and (b) GaAs nanocrystals as a function of nanocrystal radius, calculated using TB wave function and calculated by effective mass approximation.

When compared with atomistic calculation, EMA overestimates electron-hole attraction (up to 40\%) especially for small size nanocrystals. Additionally EMA shows strong $1 / R$ scaling, where atomistic results are generally described by more flat dependence [6]. This effect comes mainly from the fact that EMA functions are assumed to vanish abruptly at the boundary of quantum dot, while TB wave functions have nonzero contribution on the boundary, surface atoms.

Figure 3 explicitly shows on-site (short-range) and off-site (long-range) contributions (3) to TB calculated electron-hole Coulomb integral for InAs and GaAs nanocrystals. The on-site contribution is non-negligible only for small quantum dots $(R<1.0 \mathrm{~nm})$. For large nanocrystals the Coulomb integral is dominated by long-range monopole-monopole contribution [10], a manifestation of long-range character of the Coulomb direct interaction.

Figure 2c shows the evolution of electron-hole integral as a function of dangling bond shift for several GaAs nanocrystals. There is strong difference of magnitude of the Coulomb integrals for positive and negative shift 
values due analogous to that discussed for single particles gap and apparently confirming our speculations on stronger spatial confined states localization for negative dangling bond shifts.

Interestingly, for a given dangling bond shift sign, we find that due to the long-range character of the Coulomb interaction, electron-hole Coulomb attraction does not vary significantly with a particular choice of the shift value, especially for positive shifts, where the ground hole state is efficiently decoupled from the surface influence. Interestingly, this conclusion is also true for the short-range/on-site contribution. The on-site contribution is however a weighted average of $U_{\text {at }}$ over the quantum dot volume with electron and hole atomic charge densities (Eq. (3)), thus the surface influence is effectively smeared out.

\section{Screened electron-hole interaction}

Figure 4 shows screened Coulomb integrals calculated for (a) InAs and (b) GaAs nanocrystals as a function of nanocrystal radius calculated with quantum dot center located on different ionic species (anion, cation) and the "midpoint". Due to the long-range character of the Coulomb direct interaction, the variation of electron-hole attraction with respect to composition details is less pronounced than that of single particle gap, with little differences even for small nanocrystals.

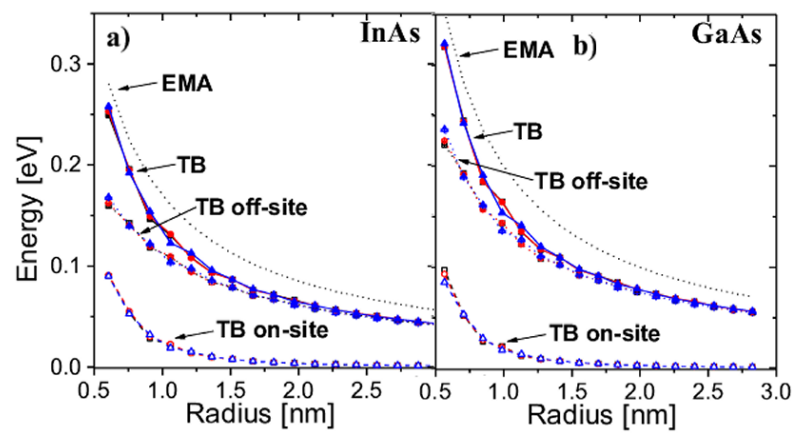

Fig. 4. Screened electron-hole $J_{e_{1} h_{1}}$ Coulomb integral calculated for (a) InAs and (b) GaAs nanocrystals as a function of nanocrystal radius calculated with quantum dot center located on different ionic species (anion, cation) and the "midpoint".

Screening affects on-site and off-site contributions on non-equal footing: off-site terms are bulk-like screened and their contribution is reduced by large factor $\left(\epsilon_{\text {bulk }}>\right.$ $10)$ compared with the unscreened case. On the contrary on-site screening is reduced $\left(\epsilon_{\mathrm{eff}} \approx 3\right)$, leading to significant overall increase of relative significance of on-site/ short-range contributions as seen in Fig. 4.

This effect may have significant consequences for other spectral quantities in particular electron-hole exchange, not considered in this paper. With different on-site terms contribution for small band gap nanocrystals (35\% compared to $28 \%$ for smallest considered InAs and GaAs nanocrystals, respectively) we speculate that this effect may affect short-range/long-range electron-hole exchange depending on nanocrystal (bulk) material, in spirit of Ref. [10]. We will leave this important subject for future work.

While the effects of lattice centering are small, there is a pronounced dependence of electron-hole interaction on the value of surface passivation/dangling bond shift (Fig. 5). Uncertainties due to the different choice of dangling bond shift are especially pronounced for small GaAs nanocrystals (on the contrary to the single particle gap, where InAs nanocrystals are the most affected). Interestingly, uncertainties due to off-site and on-site terms do not simply add up and the total electron-hole attraction variations are dominated by variations of the on-site (short-range) contribution.

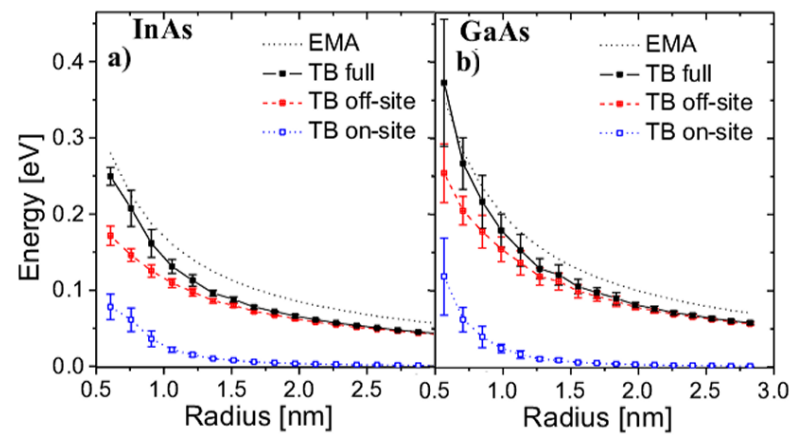

Fig. 5. Screened electron-hole $J_{e_{1} h_{1}}$ Coulomb integral calculated for (a) InAs and (b) GaAs nanocrystals as a function of nanocrystal radius calculated for extreme values of dangling bonds shift $-20 \ldots 20 \mathrm{eV}$.

Quite surprisingly, with screening effects included, the electron-hole Coulomb interaction calculated using TB approach is now much closer to that given by EMA screened value, however with the EMA values systematically overestimated for all considered nanocrystals. To analyze source of this difference in Fig. 6a we plot electron-hole Coulomb integral calculated for different diameter GaAs nanocrystals using TB approach (averaged over different surface passivations and lattice centerings), EMA and EMA with artificially increased quantum dot radius $(\Delta R=0.25 \AA)$ mimicking effects of finite potential barrier. Despite the TB curve is effectively averaged over large ensemble of systems, both EMA approaches differ systematically from atomistic approach. Thus the systematic difference between EMA and TB approaches, even though smaller than in unscreened case, cannot be attributed only to different boundary condition, but rather to effects of multi-band, multi-valley [6] coupling accounted for in TB method and neglected in straightforward effective mass approximation.

Finally in Fig. 6b we show exciton ground state energies (calculated according to Eq. (5)) for (a) InAs and (b) GaAs nanocrystals as a function of nanocrystal radius, averaged over results obtained for different, extreme val- 


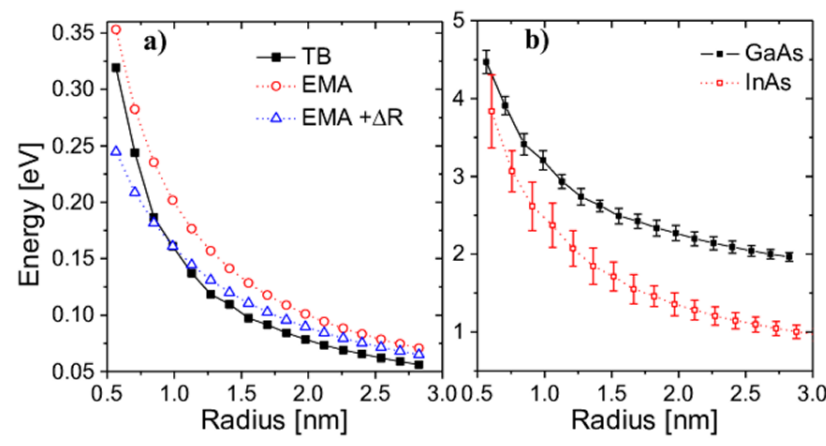

Fig. 6. (a) Screened electron-hole $J_{e_{1} h_{1}}$ Coulomb integral calculated for different diameter GaAs nanocrystals using TB approach, EMA and EMA with artificially increased quantum dot radius $(\Delta R=0.25 \AA)$. (b) Exciton ground state energies for InAs and GaAs nanocrystals as a function of nanocrystal radius, averaged over results obtained for different, extreme values of surface passivation potential $-20,20 \mathrm{eV}$ and different dot center location (anion, cation, midpoint).

ues of surface passivation potential $-20,20 \mathrm{eV}$ and different dot center location (anion, cation, midpoint). Error bars in Fig. 6b mark extreme results obtained for different calculations and are dominated by single particle gap uncertainties due to different dangling bond shifts.

\section{Conclusions}

We have studied effects of surface passivation due to dangling bond shifts and composition detail related to crystal lattice centering on single particle energies and electron-hole interaction in spherical GaAs/InAs nanocrystals. We have used atomistic, multi-band tight-binding approach and have shown that values of the single particle gap and electron and hole ground state energies are affected by certain choice of effective surface passivation potential. This effect, with possible applications to nanocrystal gap tailoring, is especially pronounced for small $(<1.5 \mathrm{~nm})$ nanocrystals originating from small bulk band gap material (InAs). We found that for the calculation of the screened Coulomb interaction, the short-range (on-site) contribution cannot be neglected and plays an important role, contributing to up to $30 \%$ of total electron-hole Coulomb attraction value in the case of small nanocrystals. For the case of screened Coulomb interaction, results obtained with simple effective mass approximation differ from the obtained within tight-binding approach and there is a systematic difference than cannot be simply attributed to different boundary conditions treatment in these two methods.

\section{Acknowledgments}

This work was supported by the Foundation for Polish Science, Homing Plus Programme co-financed by the
European Union within the European Regional Development Fund.

\section{References}

[1] L. Jacak, P. Hawrylak, A. Wojs, Quantum Dots, Springer, Berlin 1998.

[2] D. Bimberg, M. Grundmann, N.N. Ledentsov, Quantum Dot Heterostructures, Wiley, New York 1998.

[3] Nanocrystal Quantum Dots, Ed. V.I. Klimov, CRC Press, New York 2012.

[4] C. Delerue, M. Lannoo, Nanostructures: Theory and Modelling, Springer Nanosciences and Technology Series, Springer, Berlin 2004.

[5] P.E. Lippens, M. Lannoo, Phys. Rev. B 39, 10935 (1989).

[6] A. Franceschetti, A. Zunger, Phys. Rev. Lett. 78, 915 (1997).

[7] K. Leung, K.B. Whaley, Phys. Rev. B 56, 7455 (1997).

[8] J.G. Diaz, G.W. Bryant, Phys. Rev. B 73, 075329 (2006).

[9] J.-W. Luo, A. Franceschetti, A. Zunger, Phys. Rev. B 78, 035306 (2008).

[10] A. Franceschetti, L.W. Wang, H. Fu, A. Zunger, Phys. Rev. B 58, 13367 (1998).

[11] Z. Lin, A. Franceschetti, M.T. Lusk, ACS Nano 5, 2503 (2011).

[12] J.M. Jancu, R. Scholz, F. Beltram, F. Bassani, Phys. Rev. B 57, 6493 (1998).

[13] M. Zielinski, M. Korkusinski, P. Hawrylak, Phys. Rev. B 81, 085301 (2010).

[14] D.J. Chadi, Phys. Rev. B 16, 790 (1977).

[15] Z. Zhou, L. Brus, R. Friesner, Nanoletters 3, 163 (2003).

[16] X. Huang, E. Lindgren, J.R. Chelikowsky, Phys. Rev. B 71, 165328 (2005).

[17] G.W. Bryant, W. Jaskólski, J. Phys. Chem. B 109, 19650 (2005).

[18] G.W. Bryant, J. Comput. Theor. Nanosci. 6, 1 (2009).

[19] S. Lee, F. Oyafuso, P. von Allmen, G. Klimeck, Phys. Rev. B 69, 045316 (2004).

[20] S. Schulz, S. Schumacher, G. Czycholl, Phys. Rev. B 73, 245327 (2006).

[21] S. Lee, L. Jönsson, J.W. Wilkins, G.W. Bryant, G. Klimeck, Phys. Rev. B 63, 195318 (2001).

[22] C. Delerue, M. Lannoo, G. Allan, Phys. Rev. B 56, 15306 (1997).

[23] G. Allan, C. Delerue, M. Lannoo, E. Martin, Phys. Rev. B 52, 11982 (1995).

[24] C. Delerue, M. Lannoo, G. Allan, Phys. Rev. Lett. 84, 2457 (1999).

[25] A. Franceschetti, M.C. Troparevsky, Phys. Rev. B 72, 165311 (2005).

[26] M. Cardona, Phys. Status Solidi B 118, 463 (1983).

[27] L. Brus, J. Phys. Chem. 90, 2555 (1986). 